\title{
BMJ Open Common hand and wrist conditions: creation of UK research priorities defined by a James Lind Alliance Priority Setting Partnership
}

\author{
Alexia Karantana, ${ }^{1}$ Tim Davis, ${ }^{2}$ Donna Kennedy, ${ }^{3}$ Debbie Larson, ${ }^{4}$ Dominic Furniss, ${ }^{5}$ \\ Douglas J Grindlay, ${ }^{6}$ Katherine Cowan, ${ }^{7}$ Grey Giddins, ${ }^{8}$ Abhilash Jain (1) , ${ }^{9}$ \\ Ryan W Trickett (D) ${ }^{10}$
}

To cite: Karantana A, Davis T, Kennedy $\mathrm{D}$, et al. Common hand and wrist conditions: creation of UK research priorities defined by a James Lind Alliance Priority Setting Partnership. BMJ Open 2021;11:e044207. doi:10.1136/ bmjopen-2020-044207

- Prepublication history and additional material for this paper are available online. To view these files, please visit the journal online (http://dx.doi. org/10.1136/bmjopen-2020044207).

Received 01 September 2020 Revised 25 February 2021 Accepted 01 March 2021
Check for updates

(C) Author(s) (or their employer(s)) 2021. Re-use permitted under CC BY-NC. No commercial re-use. See rights and permissions. Published by BMJ.

For numbered affiliations see end of article.

Correspondence to Professor Abhilash Jain; ajainuk@aol.com

\section{ABSTRACT}

Objective Prioritisation of important treatment uncertainties for 'Common Conditions Affecting the Hand and Wrist' via a UK-based James Lind Alliance Priority Setting Partnership.

Setting This process was funded by a national charitable organisation and based in the UK.

Participants Anyone with experience of common conditions affecting the adult hand and wrist, including patients, carers and healthcare professionals. All treatment modalities delivered by a hand specialist, including therapists, surgeons or other allied professionals, were considered.

Interventions Established James Lind Alliance Priority Setting Partnership methods were employed.

Electronic and paper questionnaires identified potential uncertainties. These were subsequently confirmed using relevant, up-to-date systematic reviews. A final list of top 10 research uncertainties was developed via a faceto-face workshop with representation from patients and clinicians. Impact of research was sought by surveying hand clinicians electronically.

Outcome measures The survey responses and prioritisation—both survey and workshop based.

Results There were 889 individually submitted questions from the initial survey, refined to 59 uncertainties across 32 themes. Eight additional uncertainties were added from published literature before prioritisation by 261 participants and the workshop allowed the final top 10 list to be finalised. The top 10 has so far contributed to the award of over $£ 3.8$ million of competitively awarded funding.

Conclusions The Common Conditions in the Hand and Wrist Priority Setting Partnership identified important research questions and has allowed research funders to identify grant applications which are important to both patients and clinicians

\section{INTRODUCTION}

Hand surgery is a comparatively small subspecialty sitting under the auspices of the parent specialties of orthopaedics and plastic surgery. Elective hand conditions and hand injuries are extremely common. Between
Strengths and limitations of this study

- This Priority Setting Partnership directly involved patients and front-line clinicians throughout and is both inclusive and representative of the common demographic of hand pathology in the UK.

- Methodology strictly adhered to the well-established protocols developed by the James Lind Alliance.

- The scope of the priority setting process was broad, reporting on a wide variety of conditions affecting the adult hand.

- Conditions affecting the child's hand or rarer conditions affecting the adult hand were not included.

- Research gaps identified regarding pathophysiology, pathoanatomy, epidemiology and diagnosis were excluded from the results, in line with the James Lind Alliance methodology focusing on treatment uncertainties.

$3.7 \%$ and $5.7 \%$ of the UK population have carpal tunnel syndrome, for which over 50 000 operations were required in $2013 / 2014$. $^{1}$ Almost 20\% of emergency department attendances are for injuries to the hand. ${ }^{2}$ Although most common hand conditions are rarely life or limb threatening, they represent a significant burden of morbidity to the individual and society in general.

Historically, the priorities of researchers and academics do not necessarily align well with those of patients and front-line clinicians. $^{3-5}$ Patient and public involvement (PPI) in research has emerged as a method of addressing this imbalance. ${ }^{6}$ The National Institute of Health Research (NIHR) stipulate that PPI should form a key part of the design and development of large-scale trials, with patients not only consulted, but also routinely represented in trial design and management groups. 
The James Lind Alliance (JLA—www.jla.nihr.ac.uk) is a non-profit making initiative serving to bring together patients, carers and front-line clinicians to identify and prioritise research uncertainties in particular fields of medicine through a Priority Setting Partnership (PSP). ${ }^{7}$ A PSP highlights those research topics which are most important to patients and clinicians alike. Over 100 PSPs have been completed with many more in progress in the UK and overseas. The methodology is well established and used across healthcare fields as diverse as joint replacement surgery, cleft lip and palate, multiple sclerosis and eczema. ${ }^{8}$

\section{OBJECTIVE}

The primary aim of this work was to determine the top research priorities for common conditions affecting the adult hand and wrist. Secondarily, we aimed to assess how the identification and dissemination of these priorities has contributed to the subsequent funding success of high-quality multicentre research in hand conditions, including randomised controlled trials (RCTs).

\section{METHODS}

The 'Common Conditions of the Hand and Wrist' PSP commenced in March 2016 with the final priorities presented in November 2017. The PSP methodology used had been established by the JLA, and comprised a combination of online surveys, working group meetings and consensus workshops. ${ }^{9}$

The PSP steering group (SG) consisted of clinician and patient representatives. Patient representatives $(n=5)$ were sourced via the practices of the clinical members of the SG. Purposive sampling was considered when inviting patients to participate, to ensure participants with experience across a spectrum of hand and wrist pathology were represented. Eight clinicians were actively recruited to the SG under the guidance of the British Society for Surgery of the Hand (BSSH) Research Committee with a clinical lead (RWT) appointed to direct the process. There were six hand surgeons from the breadth of the specialty, and two hand therapists. An information specialist (DJG) from the Centre for Evidence Based Hand Surgery (CEBHS) was recruited to design and perform the literature searches and inform the uncertainty verification phases of the PSP.

The PSP was overseen by a senior JLA adviser (KC) and had administrative support from the NIHR Oxford Biomedical Research Centre. The JLA adviser facilitated and ensured balanced representation for all stakeholders throughout the PSP.

The SG convened on 12 occasions, 3 in person and 9 via teleconference. A minimum quota of patient and clinical representatives was required to attend to ratify decision making.

\section{Scope}

The wide spectrum of disease and trauma affecting the hand and wrist required a PSP with a broad scope. The
SG defined the scope as "common conditions that may require the input of a hand specialist, whether a therapist or a surgeon'. For example, traumatic brain injury while seemingly out of scope, may cause profound loss of hand function and result in the need for hand surgery and/ or hand therapy. Therefore, brain injury was included. In contrast, while chronic skin conditions such as eczema can likewise profoundly impact function of the hand, eczema is not routinely treated by a 'hand specialist', and thus excluded. Furthermore, the scope included those conditions routinely treated by non-surgical methods. It excluded conditions affecting exclusively the elbow with no distant anatomical effects on the hand, such as tennis or golfer's elbow.

In line with the JLA methodology at the time, ${ }^{9}$ the scope of the PSP was limited to identifying research uncertainties concerning the outcomes of treatment intervention, rather than the basic science or disease epidemiology. However, it became apparent from an early stage that the causes and natural history of many common hand conditions are poorly understood. Such 'out-of-scope' data were not discarded but archived to guide future basic science and epidemiology studies.

A wider Stakeholder Partnership was established to contribute questions to, publicise and disseminate the PSP, including patient groups, professional organisations and networks (see online supplemental material 1).

\section{Initial survey}

Parallel questionnaires for both patients and carers, vs clinicians were distributed through the Partnership. Surveys were made available through the SG's and Partner organisations' networks in paper form, and online via the PSP website. Social media and email campaigns were used to further publicise the surveys, which were active for 5 months.

Demographic data, including age, gender, occupation (for clinicians) and hand condition (for patients/carers), were collected from survey participants. The principle questions were:

Patient/Carer: What question(s) about the management of your hand or wrist condition(s) would you like to see answered by future research?

Clinician: What question(s) about the management of Common Conditions Affecting the Hand and Wrist would you like to see answered by future research?

Respondents were each allowed to submit up to five uncertainties or questions and given the opportunity to consent to further involvement in the PSP by providing their contact details.

\section{Uncertainty generation}

The submitted 'questions' were collated and out-ofscope submissions excluded (retaining items suitable for future review). Submissions were qualitatively analysed in a stepwise manner: reading and rereading each submission, coding each submission and combining similar 
codes into broader research 'uncertainties'. This process was completed by a single author (RWT) who carefully considered the background of the respondent (clinician vs patient/carer), and the patients' diagnosis to best determine the intention and meaning of the submitted question. Where submitted questions were considered similar, they were combined into a single uncertainty. The coding system was based on the CEBHS Hand Systematic Review (HandSRev-A comprehensive database of systematic reviews concerning topics relevant to the hand) taxonomy. ${ }^{10}$ Whenever possible, uncertainties were framed using the PICO format-Patient/Population, Intervention, Comparator and Outcome. ${ }^{11}$

Each derived research uncertainty was further reviewed and ratified by the SG, to ensure that it was broadly representative and easy to understand. The patient members of the SG separately reviewed each derived uncertainty and compared it to the original donor submission, to ensure that the uncertainty was written in lay language and the intention and meaning of the original submission had not been lost.

\section{Review of published literature and uncertainty verification}

The breadth of the PSP scope made both the literature review and uncertainty verification particularly challenging. As a result, a novel and rigorous method was developed to ensure the full breadth of eligible literature was reviewed, and that the uncertainties collected from the surveys and those extracted from the literature were checked in a scientific and reproducible way which minimised bias. This process involved the entire SG and is reported separately. ${ }^{12}$

In summary, research recommendations were extracted from relevant published Cochrane Reviews, National Institute for Health and Care Excellence guidance and Scottish Intercollegiate Guidelines Network guidelines, as well as published protocols for unpublished systematic reviews. There was no imposed time limit for inclusion of reviews, guidelines, or protocols to ensure maximum coverage of research recommendations derived from methodologically high-quality publications.

Each review, guideline and protocol was reviewed by two SG clinician members. Research recommendations were independently extracted. Differences in data extraction between reviewers were resolved via discussion. These 'extracted' uncertainties were added to those derived from the patient/carer and clinician surveys.
Uncertainty verification confirmed that stakeholder submitted research questions and extracted uncertainties were indeed unresolved uncertainties which had not already been resolved by published research in peerreviewed journals. In this PSP, the taxonomy from the HandSRev database ${ }^{10}$ was employed to map uncertainties by topic. Uncertainties were then aligned with the relevant published systematic reviews (published after 2011) collated in the HandSRev database. All systematic reviews (Cochrane and non-Cochrane) were included in the verification process regardless of methodological quality.

Mapped uncertainties were checked against the relevant systematic reviews to ensure that they had not been previously answered by original research or meta-analyses. Verification was undertaken by the clinical members of the SG. If an uncertainty was confirmed to persist, the reason for the persistent uncertainty was recorded (table 1).

All verified uncertainties were ratified for scope and content by all SG members prior to entering the prioritisation process.

\section{Interim prioritisation}

Interim prioritisation of the verified uncertainties was by an online survey made freely available via the PSP website and distributed personally to respondents to the initial patient/carer and clinician surveys who had provided contact information. The survey was open for 1 month and publicised in an identical manner to the initial patient and clinician surveys to the same group of participants.

Each survey respondent was given equal weighting in the interim prioritisation regardless of background. The survey was completed in three stages: Respondents were asked to short-list all questions considered important to them; then to select a Top ten from their shortlist; and finally, to rank their top 10 . The top 10 for each respondent was reverse scored and totals for clinicians and patient/carers were summated separately, creating separate prioritisation lists for each group. These two lists were subsequently combined to ensure equal influence from both groups.

\section{Final workshop}

The final 1-day consensus PSP workshop involved clinicians and patients purposefully sampled from the SG and survey respondents. It was facilitated by three JLA advisors. The goal was to agree a prioritised list of the top

\begin{tabular}{ll}
\hline Table 1 & Reasons recorded for persistent uncertainty \\
\hline & Available evidence supporting uncertainty \\
\hline A & No systematic review available. \\
B & Systematic review available, but authors did not find any randomised controlled trials in the literature. \\
C & Systematic review available, some randomised controlled trials found, but no formal meta-analysis performed. \\
D & Systematic review with meta-analysis available, uncertainty persists. \\
E & Cochrane systematic review with meta-analysis performed, results inclusive. \\
\hline
\end{tabular}




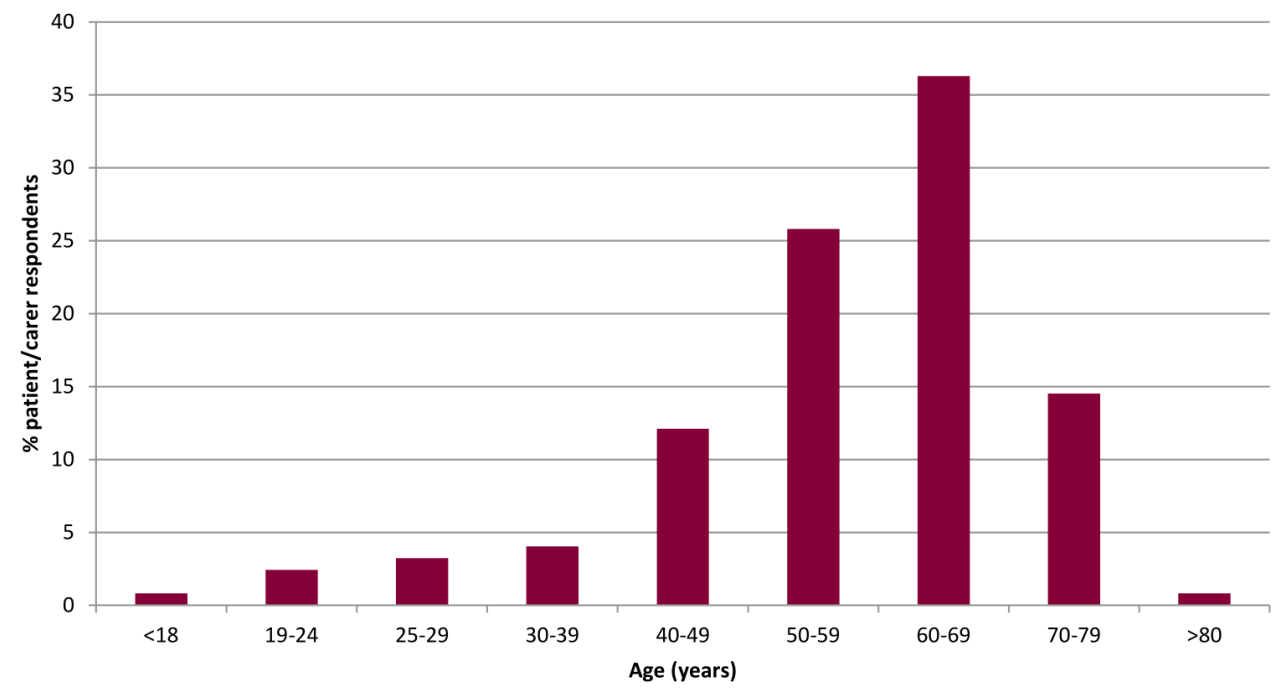

Figure 1 Age distribution of patient participants.

10 uncertainties. All potential competing interests were declared by attendees prior to the workshop.

An initial preworkshop ranking exercise was completed by each participant, forming the start point of the discussions. The workshop followed the JLA methodology of an adapted Nominal Group Technique which enables all participants to have an equal influence on the process. ${ }^{13}$ It was made clear to all participants that views based on lived experience and professional knowledge were of equal value to the discussion. Through a series of small group sessions and discussion involving the whole group, a final prioritised list was agreed. The final list was disseminated to the wider Partnership and to the public.

\section{Patient and public involvement}

The entirety of this work involved patients throughout, in line with the JLA objectives.

\section{Impact}

Following dissemination of the PSP results, the impact on the hand surgery community was assessed via a simple on-line survey. All members of the BSSH were asked to describe any research grant applications or any other research activity that was either inspired by or used the results of the PSP in any fashion. Respondents were contacted to ascertain the exact details of the impact of the PSP on their research.

\section{RESULTS}

Following removal of incomplete submissions, the initial parallel patient/carer and clinician surveys produced 889 individually submitted questions: 120 patients and 4 carers submitted 359 questions, and 152 clinicians submitted 529 questions. Most respondents were from England, but responses were received from across the UK. Many respondents were female $(74 \%)$ with a range of ages represented (figure 1).

In general, patients had undergone the full spectrum of non-surgical and surgical treatments, and many respondents had experience of multiple treatments (figure 2).

The clinician responses were characterised by type of practice. Surgeons and therapists were categorised as having a 'pure hand practice' if they spent more than two thirds of their time caring for hand conditions. Clinician respondents were most commonly hand surgeons with an orthopaedic background (table 2). The geographical distribution of clinicians reflected the distribution of specialist hand surgery units across the UK, with more

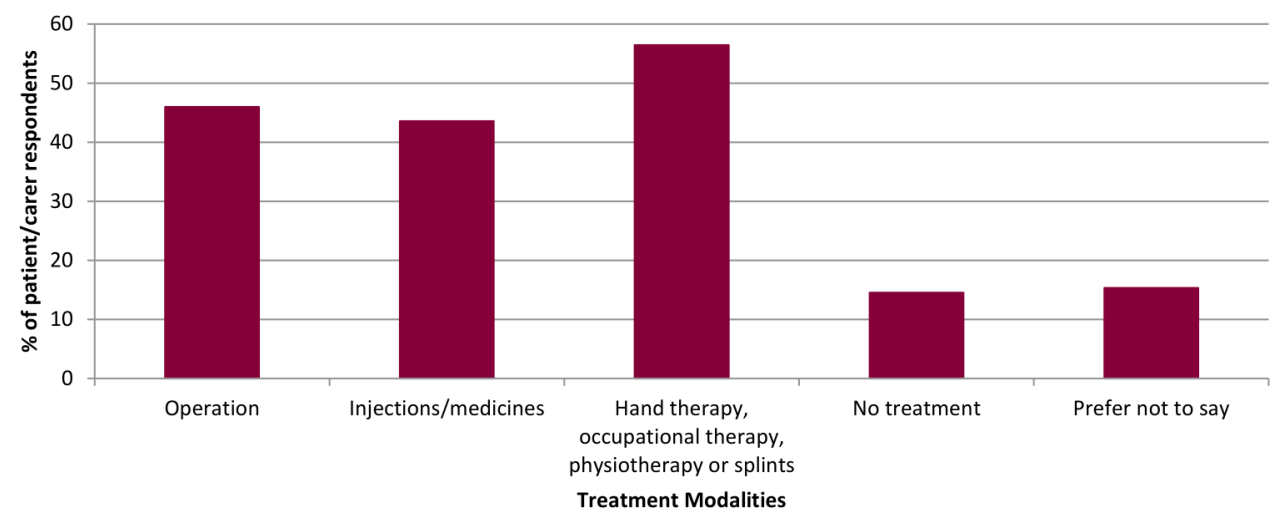

Figure 2 Treatment modalities experienced by patients. 


\begin{tabular}{lr}
\hline Table 2 Background of clinician respondents & \\
\hline Occupation/background & N \\
\hline Hand surgeon-orthopaedic background & 42 \\
\hline Hand surgeon-plastic surgery background & 15 \\
\hline Hand therapy-occupational therapy background & 18 \\
\hline Hand therapy-physiotherapy background & 10 \\
\hline Occupational therapist & 6 \\
\hline Physiotherapist & 10 \\
\hline Nurse & 3 \\
\hline Orthopaedic surgeon & 5 \\
\hline Plastic surgeon & 5 \\
\hline Other doctor operating on hand or wrist conditions & 1 \\
Other doctor treating patients with hand problems & 19 \\
\hline Other & 13 \\
\hline Prefer not to say & 5 \\
\hline Total & 152 \\
\hline
\end{tabular}

respondents based in England (81\%) than Wales (9\%), Scotland $(5 \%)$ or Northern Ireland (2\%).

Overall, the most commonly identified themes were Dupuytren's disease, osteoarthritis and inflammatory arthropathy (figure 3). This probably reflected the high activity of Partner Patient Groups in these areas. There was a wide spectrum of submissions covering the many facets of common hand practice.

The conversion of raw submissions into potential research uncertainties was performed by a single author (RWT) using the qualitative framework described in the methods. This allowed a full understanding of the range of submissions and facilitated the aggregation of similar submissions under a single uncertainty. The process was lengthy, highly subjective and often involved contextual interpretation of the submission in light of other factors concerning the individual. For example, a patient submission was 'What exercises or massages might help?' When considered alongside the patients' diagnosis of Dupuytren's Disease, this converted into the uncertainty 'What non-surgical treatments have benefits over surgery in the treatment of Dupuytren's disease?' Care was taken to ensure that the interpreted original intention of the submitter was not missed or ignored when creating uncertainties and this was confirmed by the patient members of the SG by discussion.

The 889 survey submissions were converted into a total of 59 derived potential research uncertainties (figure 3). (The full list of submissions, their categorisation and ultimate conversion is available at bit.ly/bssh_jla and via the JLA website (www.jla.nihr.ac.uk).) A total of 16 Guidelines, 44 Cochrane reviews and 17 Cochrane review protocols were assessed for additional published uncertainties. A further eight uncertainties were added to the long list for verification (figure 4). These are available in full at bit.ly/bssh_jla, or via the JLA website (www.jla.nihr.ac.uk).

All potential uncertainties were verified to be persistent uncertainties as per the JLA definition, forming the long list for interim prioritisation. The longlist was finalised on 21 February 2017 (see online supplemental material 2).

\section{Interim prioritisation}

A total of 261 participants completed the interim prioritisation survey. The majority of respondents were clinicians $(59 \%)$ rather than patients/carers $(41 \%)$.

Rankings were calculated separately for the patient/ carer and clinicians' responses and subsequently combined to give an overall ranking as per the methods described above. This ranking was reviewed by the SG to determine how many uncertainties should be taken

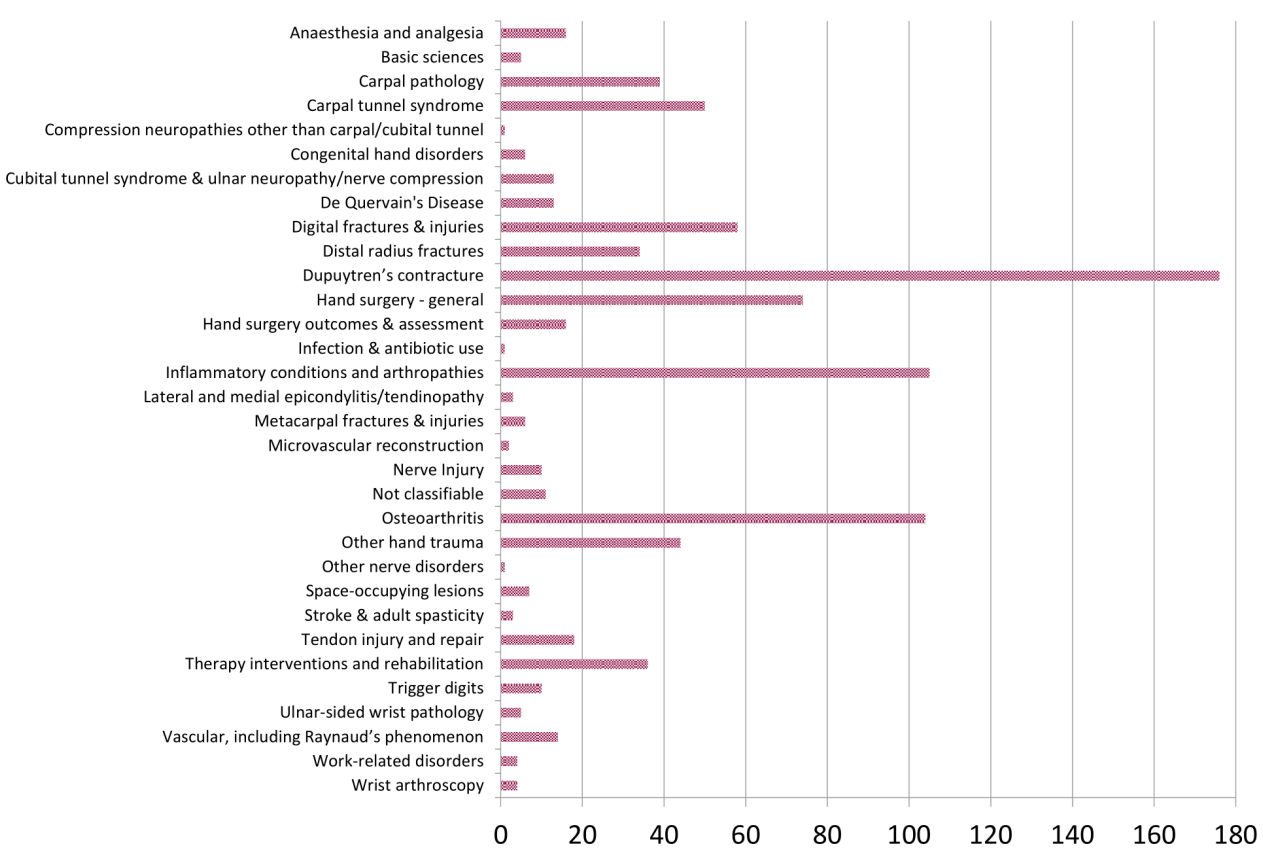

Figure 3 Themes identified from initial survey submissions (prior to removal of out-of-scope submissions). 


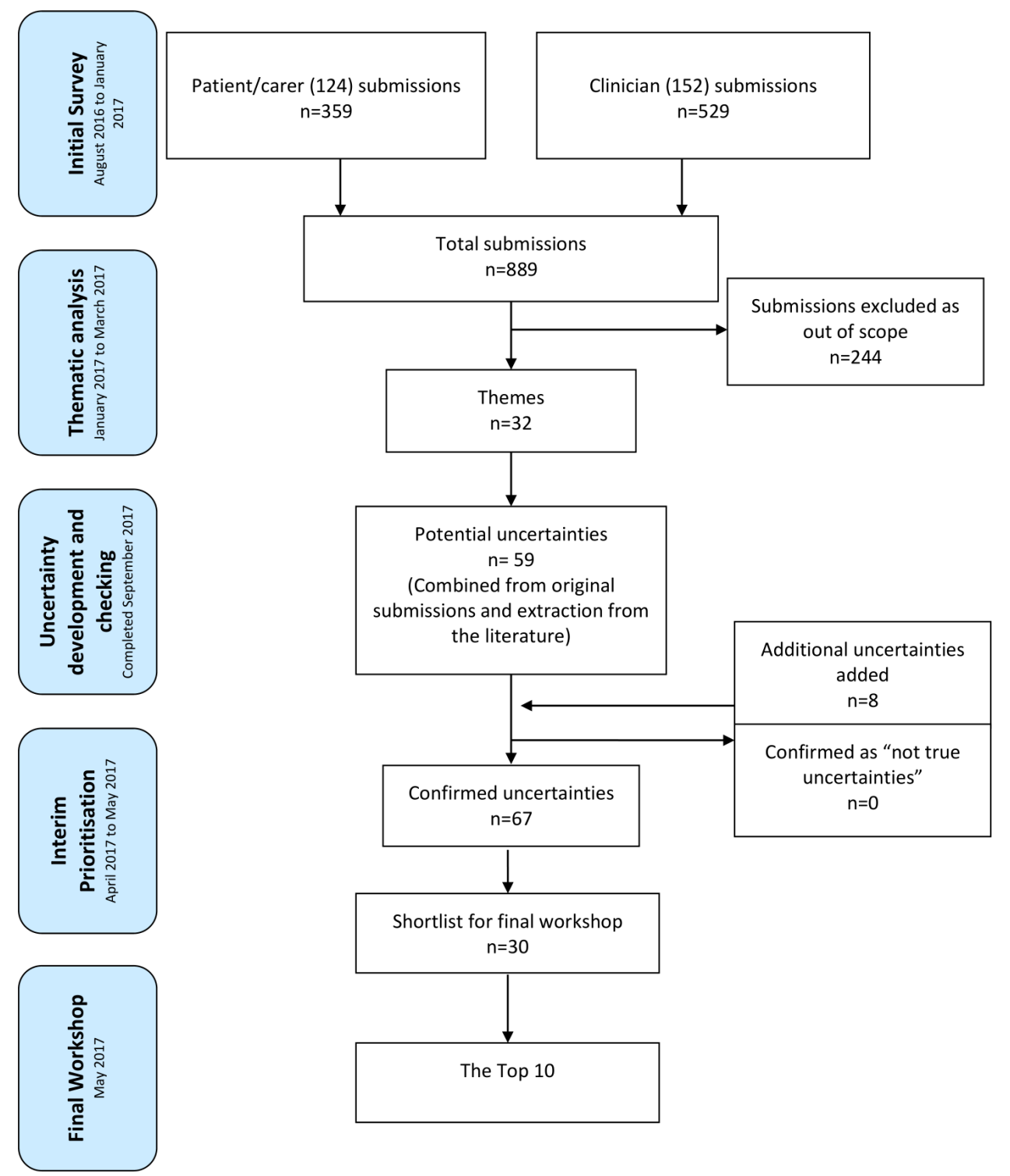

Figure 4 Flow chart of PSP process. PSP, Priority Setting Partnership.

forwards to the final workshop. The SG elected to bring the top 30 uncertainties to the final consensus workshop. This is the maximum number recommended by the JLA methodology ${ }^{9}$ and allowed inclusion of each item in the patient/carer and clinician's respective top ten.

\section{Final workshop}

The final workshop was attended by 12 clinical members (four orthopaedic hand surgeons, four plastic hand surgeons, two occupational therapy hand therapists and two physiotherapy hand therapists) and eight patient participants. In addition to the 20 participants, three facilitators and five observers also attended the final workshop. Observers did not participate in the group discussions or influence the decisions made but assisted in guiding the discussion. Facilitators were provided by the JLA and had experience of steering discussions during PSP workshops. Unanimous agreement was reached on the top 10 uncertainties. Due to the breadth of conditions represented in the short listed 30 uncertainties and the final top 10, the final workshop participants elected to not rank the top 10 As each of the top ten represented a different diagnosis, this ensured equity across conditions. The Top ten, listed alphabetically, were as follows

- Can scar/fibrosis formation be manipulated to improve results following hand surgery/trauma?

- In patients with Dupuytren's disease, what techniques give the best results in terms of function, recurrence and cost?

- In the treatment of common hand conditions, such as peripheral nerve compression syndromes (eg, carpal tunnel syndrome), ganglia or arthritis of the fingers/ thumb/wrist, do surgical interventions have a demonstrable benefit in patient reported outcome when compared with non-surgical methods or placebo (sham) surgery?

- Regarding patient and cost benefits, which interventions (eg, movement preserving surgeries such as joint or cartilage replacement, fusion operations 
permanently stiffening the joint and novel therapies) give the best results in the treatment of painful joints in the hand/wrist?

- What are the most effective non-surgical methods for treating early arthritis in the hand and fingers?

- What interventions/treatments will have the most positive effect following nerve injury?

- What methods are most accurate, user friendly and demonstrate the best clinical utility in measuring patient-reported outcomes in common hand conditions?

- Which hand therapy techniques enable the most efficient return to full function following surgery or injury?

- Which hand/finger/thumb injuries would benefit from surgical intervention over hand therapy or no formal treatment, considering both functional outcome and societal cost?

- Which patients with acute ligament injuries to the wrist or chronic wrist/distal radio-ulnar joint (the joint on the little finger side of the wrist) instability benefit from surgical treatment rather than from nonsurgical methods?

\section{Impact}

The impact survey sought to determine how the PSP had influenced current and future research studies. A simple survey concerning utilisation of the PSP findings was distributed to $979 \mathrm{BSSH}$ full and associate members. Only five responses were received. One response described a completed NIHR RCT, which had been funded and commenced prior to the PSP completion. This response was therefore excluded. There were three successful NIHR funding applications for interventional trials which had incorporated the PSP results to strengthen their applications. The three successful NIHR grants were each for between $£ 1.21$ million and $£ 1.28$ million and addressed uncertainties 8 and $52,{ }^{14}$ uncertainties 38 and $59,{ }^{15}$ and uncertainties 23 and $50^{16}$ (online supplemental material 2). A further group has been awarded £647 270 by the Medical Research Council for an experimental medicine study in thumb osteoarthritis (uncertainties 31 and 34, online supplemental material 2). A competitive research grant of $£ 65000$ was awarded to perform patient and public and clinician pump-priming work (uncertainties 7, 26, 29, 31, 34, online supplemental material 2). No responses were received concerning smaller regional or local research projects or ideas that would have increased this total.

\section{DISCUSSION}

The prime value of a PSP is helping align the mismatch between the research priorities of clinicians and patients/ carers and researchers. In this broad themed PSP, the scope has allowed patients/carers and clinicians with potentially completely disparate views and priorities to contribute to the process and establish the consensus of a top 10, on which to focus future research.

While research priority setting for musculoskeletal conditions is reported in the literature, this is the first priority setting process to be performed specifically for common conditions affecting the hand. ${ }^{17}$ Priority setting in broken bones of the upper limb in people over $50{ }^{18}$ broken bones in older people, ${ }^{19}$ cellulitis $^{20}$ and life after stroke $^{21}$ all have priorities that may be relevant to the hand. However, any such uncertainties will only relate to a small number of the hand disorders which present to hand clinicians. In contrast this PSP of "common conditions affecting the hand and wrist" considered the whole breadth of hand disorders in adults. It thus provides a balanced set of uncertainties which are relevant to patients with hand and wrist disorders, and their carers, and are faced by hand clinicians on a daily basis.

The broad scope of the 'Common Conditions of the Hand and Wrist' PSP presented an appreciable challenge in collecting, interpreting, and processing survey submissions as well as extracting and verifying all uncertainties.

The establishment of the wider Partnership required a wide review of all stakeholders. Unlike a single diagnosis PSP, there was no single patient or clinician interest group that covered the whole PSP scope and there was a risk of the members of the few single disease interest groups (ie, the Dupuytren's Society) flooding the initial survey with uncertainties relevant to their specific diagnosis. Also, it was difficult to avoid presenting a list of 'What is the best treatment for ....?' type uncertainties, with each considering a different diagnosis. This novel phenomenon termed the 'Hierarchy of Vagueness', proposed during this PSP, is best highlighted by the following example (not based on an original submission):

Original clinician submission: 'Is the XYZ plate better than the ABC external fixator in the treatment of highly comminuted wrist fractures in young adults?'

Depending on the level of detail desired in the eventual uncertainty, this question could be converted to any of the following:

- Is the XYZ plate better than the ABC external fixator in the treatment of highly comminuted wrist fractures in young adults?

- Is a plate better than an external fixator in the treatment of highly comminuted wrist fractures in young adults?

- What is the best operation for highly comminuted wrist fractures in young adults?

- What is the best operation for highly comminuted wrist fractures?

- What is the best operation for a broken wrist?

- What is the best treatment for a broken wrist?

Often a balance was sought in order to preserve the broad nature of the PSP, maintain the original intention of the submitter, and create a manageable, relevant list of uncertainties.

There were further difficulties in extracting published uncertainties and verifying potential uncertainties. There 
was no published guidance for uncertainty extraction in the JLA guidebook or from previous PSPs. The broad scope of this PSP required an extremely large and thorough literature search. The details of this challenge, alongside a protocol to standardise uncertainty extraction and checking for future PSPs are described in detail elsewhere. $^{12}$

While the surveys were open, interim analysis suggested that the majority of respondents were older English, Caucasian women, which probably skewed the patient/ carer responses towards non-traumatic conditions and away from injury, which usually occurs in men and younger people. The SG made significant efforts during the second half of the survey period to actively engage a greater number of participants from under-represented groups. This included targeted invitations to patients directly in hand clinics as well as Partner invitations to organisations representing minority groups in the community.

On closure of the surveys, the balance had improved but remained biased. The clinical members of the SG commented that, although there was an observed skew in survey participant demographics, this reflected the common patient profile seen in hand clinics. It also reflected the more active involvement of some of the partner organisations, particularly the patient groups for rheumatoid arthritis and Dupuytren's disease. Both groups have large patient networks and publicised the PSP thoroughly, but represent diseases that commonly affect women and Caucasians, respectively.

Central to any PSP is the identification of the top ten treatment uncertainties. In a PSP with a broad scope, such as Common Conditions Affecting the Hand and Wrist, inevitably this prioritisation may result in the exclusion of potentially important topics. Additionally, despite attempts to engage a diverse and inclusive partnership participant base, there is always a risk of responder bias. While there are numerous traumatic conditions represented in the top 10, distal radius and scaphoid fractures both highly prevalent conditions - were not prioritised for inclusion. Both conditions were included in the long list, but were not further prioritised by either patients or clinicians. There were significantly more patient responses from those with non-traumatic conditions (figure 3), potentially under-representing the importance of traumatic conditions in the hand. Furthermore, at the time of the interim prioritisation two prominent surgical trials were investigating both scaphoid fracture and distal radius fracture. This may have diminished the relative importance of prioritising future research in these conditions in the opinions of clinician respondents. As with any iterative consensus process, it is likely that if the process were repeated at a different time, with different respondents, the long list may have been prioritised differently.

There has been demonstrable research impact following the PSP. Over £3 million of competitively awarded major research funding has been awarded since the dissemination of the PSP results. We purposefully delayed the impact survey (performed February 2019) to maximise the time available to researchers to incorporate the PSP findings into their research activity. While the survey remains open, no responses were received after May 2019. Despite this, the response to the impact survey was considerably lower than expected for any potential smaller scale research projects, inclusion of which would have likely added further impact. Indeed, the BSSH have awarded a number of small scale competitive research grants to applicants quoting this PSP. Another explanation is that these smaller projects are often pilots and are not externally funded. However, we hypothesise that the PSP longlist is likely to have seeded multiple further research projects and ideas. Determining the impact of a PSP is difficult and not limited to successful funding calls. ${ }^{22}$ However, funding for research provides a quantitative assessment of the relative impact of a PSP and is easier to interpret—and apply value to-when compared with qualitatively measured impact for patients, organisations, clinicians, and academics.

\section{Author affiliations}

${ }^{1}$ Centre for Evidence Based Hand Surgery, School of Medicine, University of Nottingham, Nottingham, UK

${ }^{2}$ Trauma and Orthopaedics, Nottingham University Hospitals NHS Trust, Nottingham, UK

${ }^{3}$ Pain Research Group, Imperial College London, London, UK

${ }^{4}$ Hand Therapy, Spire Norwich Hospital, Norwich, UK

${ }^{5}$ Genetics and Epidemiology of Common Hand, University of Oxford Nuffield Department of Surgical Sciences, Oxford, UK

${ }^{6}$ Centre for Evidence Based Hand Surgery, School of Medicine, University of Nottingham, Nottingham, UK

${ }^{7}$ James Lind Alliance, Southampton, UK

${ }^{8}$ Orthopaedic Department, Royal United Hospital Bath NHS Trust, Bath, UK

${ }^{9}$ Department of Orthopaedics Rheumatology and Musculoskeletal Sciences, University of Oxford Nuffield, Oxford, UK

${ }^{10}$ Trauma and Orthopaedics, Cardiff and Vale University Health Board, Cardiff, UK

\section{Twitter Ryan W Trickett @valehandsurgery}

Acknowledgements Steering Group patient members: Christopher Delaney, Jo Horsey, David Skilton, Sheila Wade and Gillian Walton, and those clinicians and patients who volunteered their valuable time for submission of questions, responding to surveys, or participating in the final workshop. The British Society for Surgery of the Hand for funding the PSP. All our partner organisations (listed in online supplemental material 1), and particularly the British Association of Hand Therapists who contributed the input of their membership, vital in publicising this PSP.

Contributors Origin of study idea: TD, AJ. PSP design: RWT, KC. Study management: AK, TD, DK, DL, DF, DJG, KC, GG, AJ, RWT. Acquisition of data: AK, TD, DK, DL, DF, DJG, KC, GG, AJ, RWT. Analysis of data: RWT. Interpretation of data: AK, TD, DK, DL, DF, DJG, KC, GG, AJ, RWT. Drafting and revision: AK, TD, DK, DL, DF, DJG, KC, GG, AJ, RWT. Final approval: AK, TD, DK, DL, DF, DJG, KC, GG, AJ, RWT. Agreement of accountability: AK, TD, DK, DL, DF, DJG, KC, GG, AJ, RWT. All authors met the ICMJE criteria for authorship.

Funding This Priority Setting Partnership was funded in full by a grant from the British Society for Surgery of the Hand (BSSH).

Competing interests This work was funded by the British Society for Surgery of the Hand. AK, AJ, GG, DF, TD and RWT are consultant hand surgeons and members of the British Society for Surgery of the Hand. All are active in clinical and nonclinical research. KC is a senior adviser to the James Lind Alliance and coauthor and editor of the JLA Guidebook. She supports the JLA Secretariat including in recruiting and training the team of JLA advisers. She was an independently contracted adviser for this work.

Patient consent for publication Not required. 
Provenance and peer review Not commissioned; externally peer reviewed.

Data availability statement Data are available in a public, open access repository. Raw data available at http://www.jla.nihr.ac.uk/priority-setting-partnerships/ common-conditons-affecting-the-hand-and-wrist/

Supplemental material This content has been supplied by the author(s). It has not been vetted by BMJ Publishing Group Limited (BMJ) and may not have been peer-reviewed. Any opinions or recommendations discussed are solely those of the author(s) and are not endorsed by BMJ. BMJ disclaims all liability and responsibility arising from any reliance placed on the content. Where the content includes any translated material, BMJ does not warrant the accuracy and reliability of the translations (including but not limited to local regulations, clinical guidelines, terminology, drug names and drug dosages), and is not responsible for any error and/or omissions arising from translation and adaptation or otherwise.

Open access This is an open access article distributed in accordance with the Creative Commons Attribution Non Commercial (CC BY-NC 4.0) license, which permits others to distribute, remix, adapt, build upon this work non-commercially, and license their derivative works on different terms, provided the original work is properly cited, appropriate credit is given, any changes made indicated, and the use is non-commercial. See: http://creativecommons.org/licenses/by-nc/4.0/.

\section{ORCID iDs}

Abhilash Jain http://orcid.org/0000-0002-1799-5310

Ryan W Trickett http://orcid.org/0000-0002-0688-0630

\section{REFERENCES}

1 Burton CL, Chen Y, Chesterton LS, et al. Trends in the prevalence, incidence and surgical management of carpal tunnel syndrome between 1993 and 2013: an observational analysis of UK primary care records. BMJ Open 2018;8:e020166.

2 Burke FD, Dias JJ, PALOU CH, et al. Providing care for hand disorders a re-appraisal of need. $J$ Hand Surg Am 2004;29:575-9.

3 Crowe S, Fenton M, Hall M, et al. Patients', clinicians' and the research communities' priorities for treatment research: there is an important mismatch. Res Involv Engagem 2015;1:2.

4 Tallon D, Chard J, Dieppe P. Relation between agendas of the research community and the research consumer. Lancet 2000;355:2037-40.

5 Boddy K, Cowan K, Gibson A, et al. Does funded research reflect the priorities of people living with type 1 diabetes? a secondary analysis of research questions. BMJ Open 2017;7:e016540.

6 Tallon D, Chard J, Dieppe P. Consumer involvement in research is essential. BMJ 2000;320:380-1.

7 Partridge N, Scadding J. The James Lind alliance: patients and clinicians should jointly identify their priorities for clinical trials. Lancet 2004;364:1923-4.
8 Pauldine R, Beck G, Salinas J, et al. Closed-loop strategies for patient care systems. J Trauma 2008;64:S289-94.

9 JLA. The James Lind alliance guidebook, 2018. Available: http:// www.jla.nihr.ac.uk/jla-guidebook/

10 HandSRev. Centre for evidence based hand surgery. Available: https://www.nottingham.ac.uk/research/groups/cebhs/handsrev/ index.aspx

11 HandSRev. Centre for evidence based hand surgery. Available: http:// www.nottingham.ac.uk/research/groups/cebhs/handsrev/index.aspx

12 Grindlay D, Davies TRC, Kennedy D, et al. Uncertainty extraction and verification for the common conditions affecting the hand and wrist priority setting partnership: a proposed methodology for priority setting partnerships with the James Lind alliance. medRxiv 2021.

13 McMillan SS, King M, Tully MP. How to use the nominal group and Delphi techniques. Int J Clin Pharm 2016;38:655-62.

14 Research NIfH. Research award: POINT: A multi-centre randomised trial of surgery versus non-surgical splint treatment for proximal phalanx shaft finger fractures in adults [NIHR127292] 2020. Available: https://www.fundingawards.nihr.ac.uk/award/ NIHR127292

15 Research NlfH. Resesarch award: 18/37 - Repair of digital nerve injury [NIHR127807], 2020. Available: https://www.fundingawards. nihr.ac.uk/award/NIHR127807

16 Research NIfH. Research award: Hand-2: Needle fasciotomy versus limited fasciectomy for treatment of Dupuytren's contractures of fingers: a randomised non-inferiority trial with an individual participant data network meta-analysis comparison with DISC (ISRCTN18254597) to compare outcomes after collagenase injections [NIHR127393], 2020. Available: https://www. fundingawards.nihr.ac.uk/award/NIHR127393

17 Bourne AM, Johnston RV, Cyril S, et al. Scoping review of priority setting of research topics for musculoskeletal conditions. BMJ Open 2018;8:e023962.

18 Sheehan WJ, Williams MA, Paskins Z, et al. Research priorities for the management of broken bones of the upper limb in people over 50: a UK priority setting partnership with the James Lind alliance. BMJ Open 2019;9:e030028.

19 Fernandez MA, Arnel L, Gould J, et al. Research priorities in fragility fractures of the lower limb and pelvis: a UK priority setting partnership with the James Lind alliance. BMJ Open 2018;8:e023301.

20 Thomas KS, Brindle R, Chalmers JR, et al. Identifying priority areas for research into the diagnosis, treatment and prevention of cellulitis (erysipelas): results of a James Lind alliance priority setting partnership. Br J Dermatol 2017;177:541-3.

21 Pollock A, St George B, Fenton M, et al. Top ten research priorities relating to life after stroke. Lancet Neurol 2012;11:209.

22 Staley K, Crowe S, Crocker JC, et al. What happens after James Lind alliance priority setting partnerships? A qualitative study of contexts, processes and impacts. Res Involv Engagem 2020;6:41. 